\title{
Doping-free carbon nanotube optoelectronic devices
}

\author{
WANG Sheng ${ }^{*}$, ZHANG ZhiYong \& PENG LianMao* \\ Key Laboratory for the Physics and Chemistry of Nanodevices, Department of Electronics, Peking University, Beijing 100871, China
}

Received April 12, 2011; accepted July 26, 2011; published online November 26, 2011

\begin{abstract}
Semiconducting carbon nanotubes (CNTs) possess outstanding electrical and optical properties because of their special one-dimensional (1D) structure. CNTs are direct bandgap materials, which makes them ideal for use in optoelectronic devices, e.g. light emitters and light detectors. Excitons determine their light absorption and light emission processes due to the strong Coulomb interactions between electrons and holes in CNTs. In this paper, we review recent progress in CNT photodetectors, photovoltaic devices and light emitters. In particular, we focus on the doping-free CNT optoelectronic devices developed by our group in recent years.
\end{abstract}

carbon nanotube, diode, photodetector, LED, solar cell, doping-free

Citation: Wang S, Zhang Z Y, Peng L M. Doping-free carbon nanotube optoelectronic devices. Chin Sci Bull, 2012, 57: 149-156, doi: 10.1007/s11434011-4806-3

The semiconducting single walled carbon nanotube (SWCNT) is a typical direct-gap 1D material, which is suitable for the construction of nano-scale optoelectronic devices. Interest in CNT-based photoelectronics stems from the possibility of producing nano-scale light emitters and photoconductors, which can then be used in integrated multifunctional photonic circuits [1]. The CNT has good absorption across the ultraviolet-infrared (UV-IR) spectra and almost no light is reflected from CNT thin films. Semiconducting CNTs also have several unique features that are important for IR photodetectors; these include extreme IR light absorption and high room temperature mobility for an ultrafast ps-scale photoresponse [2,3]. The diameters of CNTs are tunable and multiple nanotubes with different band structures can be combined to match the solar spectrum for photovoltaic (PV) applications. CNT solar cells also provide a new choice for future environmentally clean energy sources [4,5].

The optical properties of CNTs, including light absorption and light emission, are determined by bound exciton states due to the strong Coulomb interaction between electrons and holes in the 1D system [6]. The exciton binding energy of the semiconducting SWCNT depends inversely

*Corresponding authors (email: shengwang@pku.edu.cn; lmpeng@pku.edu.cn) on the CNT diameter, and it is $\sim 0.43 \mathrm{eV}$ for a typical SWCNT $(6,5)$ with $d \sim 0.8 \mathrm{~nm}$ [7]. The exciton binding energy also depends on the dielectric environment in which the CNTs are embedded [8]. These two excitonic characteristics provide feasible methods which can be used to modulate the properties of CNT optoelectronic devices in applications. To date, many types of CNT optoelectronic device have been investigated, including light detectors, light emitters and solar cells. In this paper, we review the progress in CNT photoelectric devices over the past few years.

\section{CNT photodetectors and solar cells}

Photodetectors based on CNTs have been demonstrated in field-effect transistors (FET) [9-11], Schottky-barrier (SB) diodes [12] and p-n junctions [13,14]. In early reports on CNTFET photoconductivity, oxygen absorption and desorption was found to be an important factor for the photoresponse of CNTFETs to high-energy photons such as UV illumination [11]. The photocurrent of the CNTFET is generated by excitation, which can be used to make a polarized photoconductor [9]. The maximum internal quantum efficiency of the CNTFET photoconductor was estimated to be about $10 \%$ by a group from IBM [9]. If the Schottky 
barriers at the two source and drain contacts of the CNTFET device are different, the photovoltage can be observed [12]. Typical open-circuit photovoltages of $V_{\mathrm{OC}}=300 \mathrm{mV}$ and short-circuit currents of $I_{\mathrm{SC}}=100 \mathrm{pA}$ are generated when the device is illuminated with uniform infrared light at about $1 \mathrm{~kW} / \mathrm{cm}^{2}$. In such a device structure, the open-circuit photovoltage is independent of the gate voltage because the maximum photovoltage is determined by an internal field generated by the height difference between the two SBs [12]. However, the SB will reduce the maximum achievable photovoltage, which is limited by the CNT bandgap.

In modern optoelectronic devices, the p-n junction is the basic building block of most photodetectors, light sources and solar cells, but it is difficult to form an air-stable p-n junction using traditional doping methods [15]. It may also lead to low efficiency for induction of more scattering centers or trap states by chemical doping with organic materials [16]. A CNT p-n junction with a split gate structure was designed successfully by Lee et al. [17]. Two split gates separated by $0.5 \mu \mathrm{m}$ are used to form the p-region and the n-region in two halves of the same CNT with different electrostatic doping controls. The CNT diode using this p-n junction shows good rectifying $I-V$ characteristics and fits well with the diode equation, with ideality $n=1.2$ [17]. A device with an ideality factor of $n=1$ is also obtained when the CNT is suspended [13]. One important mechanism for multiple electron-hole pair generation was first observed in

(a)
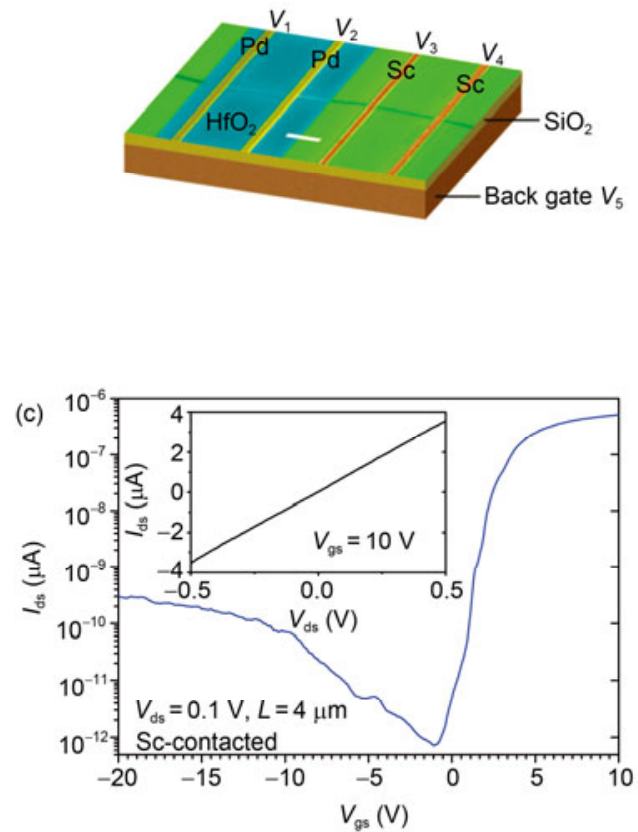

such a split gated CNT p-n junction [14]. This carrier multiplication can improve the efficiency of photovoltaic solar cells beyond the Shockley-Quiesser limit [18]. However, PV devices based on this p-n junction can only work under different gate bias controls, and are not suitable for applications because of the extra power supply requirements and the complicated fabrication process.

In the past few years, we have developed a new type of CNT diode device, the barrier-free bipolar diode (BFBD), with doping-free methods [19]. This device was first constructed in a CNT complementary metal-oxide semiconductor (CMOS) unit, as shown in Figure 1(a). The CNT device with two Pd electrodes exhibits p-type FET characteristics (Figure 1(b)). When Sc electrodes are used, the device characteristics become those of an n-type FET (Figure 1(c)). The device can function as a CMOS inverter when the two middle electrodes $\left(V_{2}, V_{3}\right)$ are connected, with $V_{\text {OUT }}=$ $V_{2}=V_{3}$ and $V_{\text {IN }}=V_{5}$. In addition to the basic function of a CNT inverter shown in Figure 1(a), we also found that the CNT segment between the $V_{2}$ and $V_{3}$ electrodes can be used to form a high-quality diode, as shown in Figure 2. Although this CNT segment is redundant for the CMOS inverter, it is not detrimental to the inverter performance, with a high voltage gain of more than 10 (Figure 1(d)).

Our CNT BFBD consists of an intrinsic semiconducting SWCNT, which is asymmetrically contacted using Pd and Sc or Y electrodes (Figure 1(a)). It has been shown that

(b)
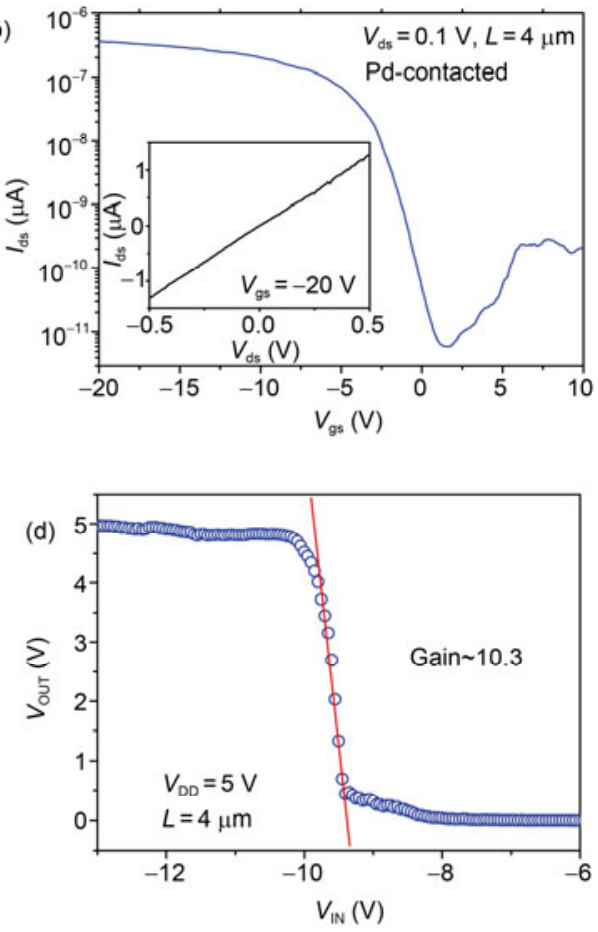

Figure 1 CNT based CMOS inverter characteristics. (a) SEM image of the device. The transfer characteristics, i.e. $I_{\mathrm{ds}}-V_{\mathrm{gs}}$ for (b) Pd-contacted CNT p-type FET (with $V_{\mathrm{ds}}=V_{1}, V_{2}=0, V_{\mathrm{gs}}=V_{5}$ ) and (c) Sc-contacted CNT n-type FET (with $V_{\mathrm{ds}}=V_{3}, V_{4}=0, V_{\mathrm{gs}}=V_{5}$ ). (d) Transfer characteristics of the CMOS inverter with $V_{\mathrm{DD}}=V_{1}, V_{4}=0, V_{2}=V_{3}=V_{\mathrm{OUT}}, V_{\mathrm{IN}}=V_{5}$, showing a voltage gain of more than 10 . The diameter of the CNT is $\sim 1.5 \mathrm{~nm}$, and all channel distances between electrodes (Pd-Pd, Pd-Sc, Sc-Sc) are $4 \mu \mathrm{m}$. The inserts in (b) and (c) are the drain $I-V$ characteristics of the FETs, showing that the contacts between CNT/Pd and $\mathrm{CNT} / \mathrm{Sc}$ are both ohmic [19]. 

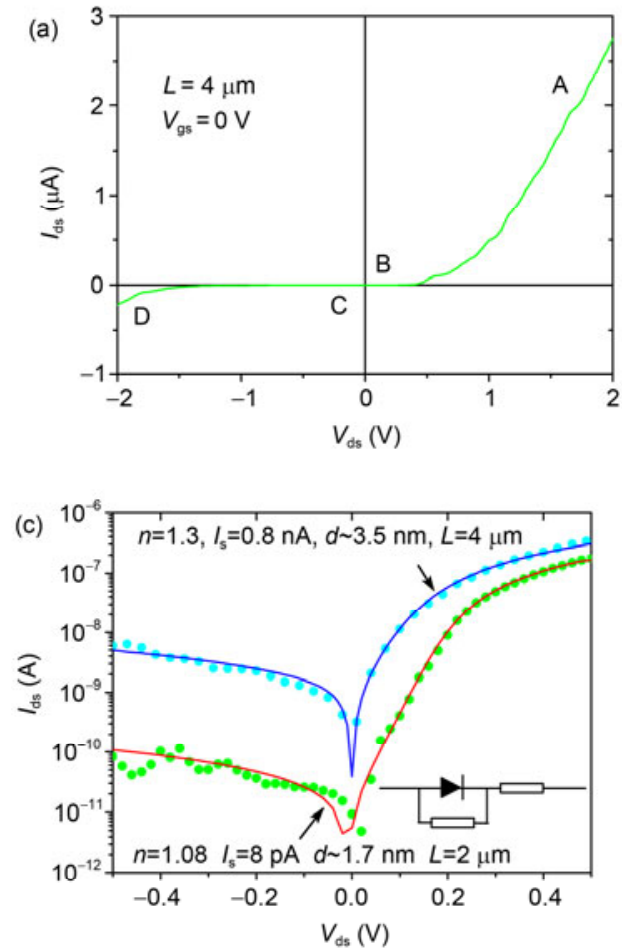

(b)
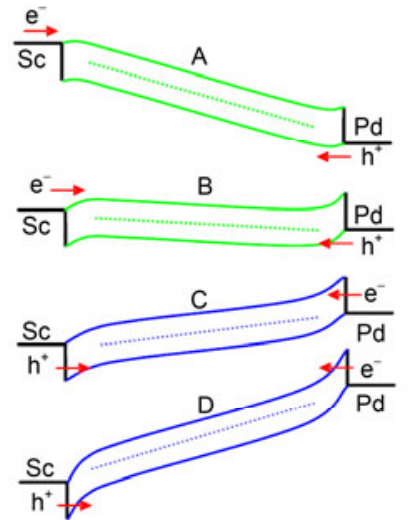

(d)

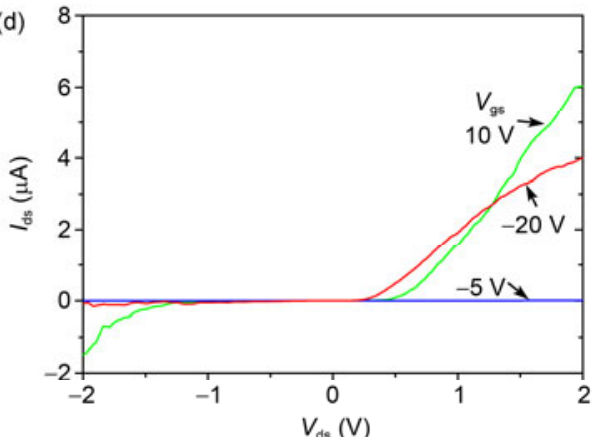

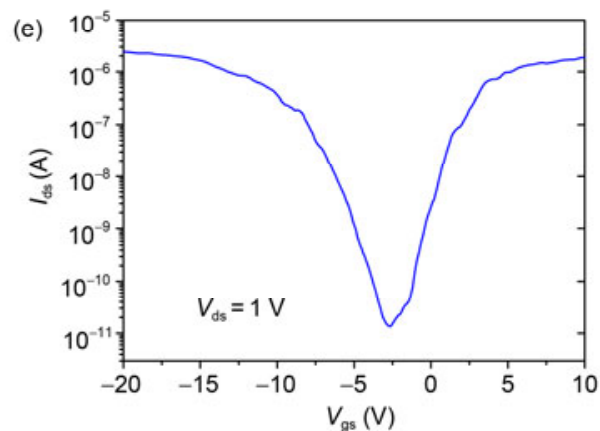

Figure 2 Characteristics of barrier-free bipolar diode. (a) $I$ - $V$ curve of a typical diode, with CNT diameter $d \sim 1.5 \mathrm{~nm}$ and length $L \sim 4 \mu \mathrm{m}$. (b) The energy band diagram of the asymmetrically contacted CNT which corresponds to the four points A, B, C, and D of (a). (c) Experimental and fitted $I-V$ curves for two CNTs with different diameters of $d \sim 1.7 \mathrm{~nm}$ and $d \sim 3.5 \mathrm{~nm}$. (d) $I-V$ characteristics for a BFBD device at three different gate voltages. (e) Gate transfer characteristics for the same diode with source-drain bias $V_{\mathrm{ds}}=1 \mathrm{~V}$, showing that the device is indeed an ambipolar FET [19].

carriers can be injected, barrier-free, into the valence band of the CNT from the Pd electrode [20], and into the conduction band from the Sc or Y electrode [21-23]. For a BFBD device with a smaller CNT diameter $d \sim 1.7 \mathrm{~nm}$ and a channel length $L=2 \mu \mathrm{m}$, we find that $n \sim 1.08$ fits well in the modified diode equation [24]: $I=\left(V-I R_{\mathrm{S}}\right) / R_{\mathrm{sh}}+I_{\mathrm{s}}[\exp (q(V-$ $\left.I R_{\mathrm{s}}\right) / n k T$ ) -1], as shown in Figure 2(b) [19]. This value is very close to the ideal factor of 1 and shows that our diode exhibits a high performance PV effect [25]. The $I-V$ characteristics of the diode (Figure 2(c) and (d)) also depend on the gate voltage $V_{\mathrm{gs}}$, providing another possibility to further improve the diode properties. The ambipolar transport under forward bias of $V=1 \mathrm{~V}$ means that both electrons and holes can be injected into the CNT channel effectively under the appropriate gate voltage. Figure 2(d) shows three distinct $I-V$ curves measured with three gate voltages: $V_{\mathrm{gs}}=10,-5$, $-20 \mathrm{~V}$. The device shows good rectification at both large positive $V_{\mathrm{gs}}=10 \mathrm{~V}$ and negative $V_{\mathrm{gs}}=-20 \mathrm{~V}$.

The response of the BFBD device with CNT $d \sim 1.5 \mathrm{~nm}$ to infrared laser illumination is shown in Figure 3(a). The three curves correspond to dark, illumination with power density of $9 \mathrm{~kW} / \mathrm{cm}^{2}$ and illumination at $45 \mathrm{~kW} / \mathrm{cm}^{2}$ [25]. The energy band diagram (Figure 3(b)) summarizes the essential physical processes of the BFBD under illumination. A built-in field is formed across the device, even in the middle region, for a device channel length $L=800 \mathrm{~nm}$, effectively separating the photo-generated electron-hole pairs in the device and resulting in photocurrent and photovoltage. 


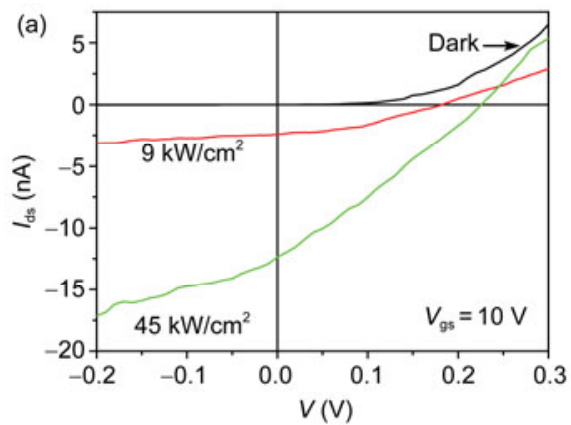

(b)

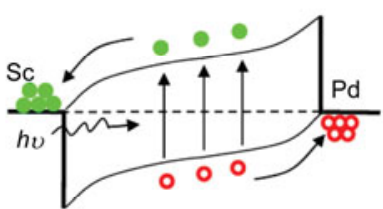

Figure 3 PV characteristics of a BFBD device. (a) $I-V$ curves measured in the dark and under illumination, corresponding to photovoltage and photocurrent with increasing light intensity. (b) Energy band diagram of the BFBD showing the process of generation and transport of photocarriers within the CNT channel [25].

Figure 4(a) shows the dependence of both $V_{\mathrm{OC}}$ and $I_{\mathrm{SC}}$ on the illumination power density. For a power density lower than $45 \mathrm{~kW} / \mathrm{cm}^{2}, I_{\mathrm{SC}}$ varies linearly with the power density, but it starts to saturate for larger light power densities. This is because the larger illumination power may lead to a higher sample temperature, which in turn results in a higher recombination rate for the photogenerated e-h pairs. $V_{\mathrm{OC}}$, on the other hand, follows a logarithmic dependence on the illumination power density for small power densities and begins to saturate at about $45 \mathrm{~kW} / \mathrm{cm}^{2}$. An open-circuit voltage of $V_{\mathrm{OC}}=0.23 \mathrm{~V}$ and a large photocurrent $I_{\mathrm{SC}}$ of more than $15 \mathrm{nA}$ can be obtained for CNT BFBD device with $d \sim 1.5 \mathrm{~nm}$. For our devices, the fill factor (FF) ranges from $0.29-0.51$ and conversion efficiency $\eta \sim(0.1 \%-0.4 \%)$ for a typical single nanotube device with low light absorption. However, the actual quantum efficiency is of the same order as that reported for other device geometries, i.e. $\eta \sim 10 \%$ [9].

The p-n junction forms the basis of most traditional solar cells. CNT solar cells can be classified as homojunction or heterojunction solar cells. One important progression is CNT solar cells based on the homojunction, in which a built-in field can be constructed to separate the photocarriers. CNT homojunction solar cells were mainly fabricated using doping methods or by forming different metal-CNT contacts in early reports of these devices $[5,13,16,26]$. The CNT photovoltaic effect based on a $\mathrm{p}-\mathrm{n}$ junction with split gate control can also be applied to solar cells, but the fabrication process is relatively complicated and the cells need an externally applied gate voltage to convert the solar power. Asymmetrical contact $\mathrm{Pd}$ and $\mathrm{Al}$ electrodes connected to CNTs via a welding method can also be used as solar cells, but it is difficult to fabricate large scale devices because of the welding process [26].

Heterojunctions between different materials and CNTs can be formed through bandgap matching. Materials such as CNT and n-type heavily doped silicon [27-29], CNT-CdSe Schottky junction solar cells [30], and CNT-GaAs heterojunctions [31] have been designed to act as photovoltaic cells. However, the efficiencies of these heterojunctionbased solar cells are still limited to within 7\% [28]. The efficiency can be improved by decreasing recombination at the interface and increasing light absorption at the junction area. CNTs have also been used as light harvesting materials or as electrodes to collect the photocarriers because of their high efficiency light absorption and excellent conductance properties [32,33].

Our doping-free CNT solar cells based on the BFBD device have some advantages for current solar cell development. The CNT-type solar cell structure is fabricated via a very simple process, and only two types of metal must be used [19]. The CNT cell is not dependent on dopants, which are used in the production of many solar cells. The doping-
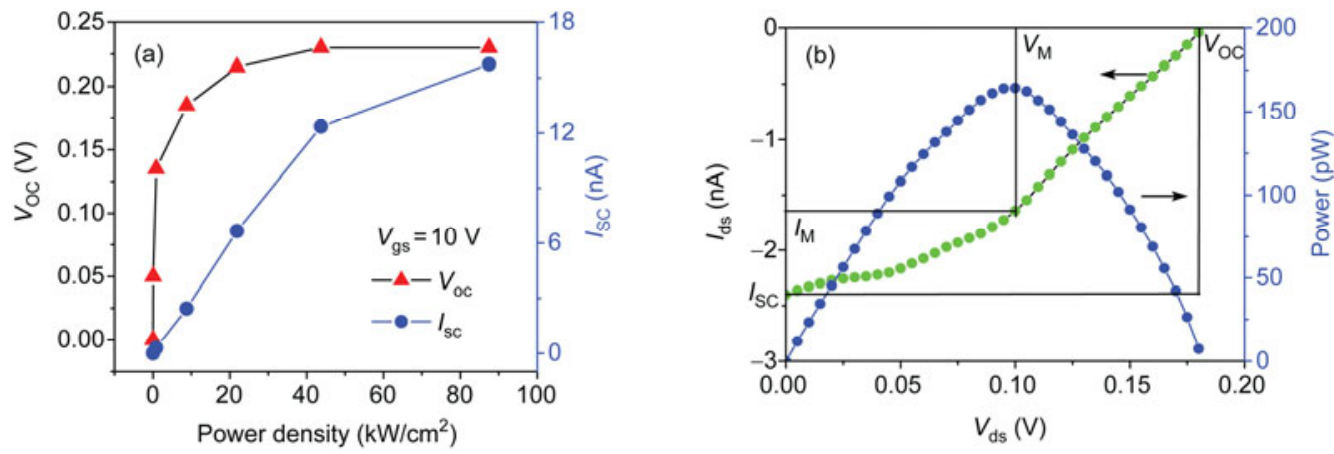

Figure 4 (a) Photocurrent and photovoltage as a function of the illumination power density of a $785 \mathrm{~nm}$ laser. (b) The fourth quadrant $I-V$ curve and power generation under $9 \mathrm{~kW} / \mathrm{cm}^{2}$ illumination [25]. 
free CNT solar cells may thus be used in high temperature or extremely low temperature environments without failures caused by dopant diffusion or dopant freezing.

Although the conversion efficiency of the single SWCNT solar cell is far lower than that of existing silicon-based solar cells, these advantages are sufficient to encourage us to improve the efficiency with new methods or designs. For example, PV efficiency can be improved by using a high purity semiconducting CNT film.

\section{CNT light emitter}

CNT light emitters have been reported based on FETs [34-37], p-n junctions with electrostatic doping methods and split gates [38], and BFBD devices with asymmetric contacts [39]. Electroluminescence (EL) from a network of CNTs has also been investigated in a FET geometry using CNT films or arrays of aligned CNTs [40-43], but the excitation mechanisms in these devices are more complicated because of the extra interactions between the CNTs. The light emission efficiency may be decreased by the presence of metallic nanotubes due to non-radiative exciton annihilation or by exciton transfer between the CNTs [43].

There are three main mechanisms of excitation in the theory of electroluminescence [44]. One mechanism is the direct ionization of impurity systems by a strong local electric field of about $10^{7} \mathrm{~V} / \mathrm{cm}$. Another is an impact excitation mechanism with a local field of $\sim 10^{5} \mathrm{~V} / \mathrm{cm}$, where the electrons or holes are accelerated under a large electric field and excite the impurity system or electrons via inelastic collisions. The final mechanism is by direct recombination of injected charge carriers (electrons and holes), which usually occurred in traditional p-n junction devices. The latter two mechanisms are the main principles of CNT EL devices.

In the previous investigations of CNT light sources, EL sources based on the CNT-FET device are the most successful and most detailed. The FET can be either the ambipolar type or the unipolar type with different contact electrodes [1,34-37]. In the ambipolar FET, electrons and holes can be injected simultaneously into the CNT channel via tunneling, and then recombine to yield polarized light at large bias and under suitable gate voltage. The FET can appear to be unipolar where only one type of carrier (e.g. holes for a p-type FET with Pd contacts) is injected into the CNT. At a large enough bias, a strong local electric field may be generated around defects to induce impact excitations because of the high kinetic energy carriers, generating electron-hole pairs and subsequently causing local light emission [35]. At the same time, another unexpected result of the use of a strong electric field in these devices is that it yields a much broader EL spectrum (150 meV) by mixing the excitonic states with those of the band-to-band continuum, which leads to spectral weight transfer from the excitonic peak to the continuum. The efficiency $\eta$ of the radiative recombination process for EL is $\sim 10^{-6}$ photons/electron-hole pair for the ambipolar FET, but it can be increased to $10^{-4}$ $10^{-3}$ for the $\mathrm{p}-\mathrm{n}$ junction diode [38].

Recently, a CNT based light emitting p-n diode was developed by a group at IBM, using the electrostatic doping technique that has been used in the investigation of CNT photoelectric devices [38]. They demonstrated the LED principle in a SWCNT for the first time. The light emission of the p-n junction LED appeared to have a narrower peak of $\sim 35 \mathrm{meV}$, and a much higher light generation efficiency. As discussed previously, the structure of this $p-n$ diode is very complex, requiring different biasing to form electron $(n-)$ and hole (p-) rich regions with reverse gate voltages. The EL mechanism in such a diode is unlike that of the conventional LED, which is a forward biased p-n junction, where carriers are injected into the recombination area mainly via thermal excitation. The IBM CNT p-n diode operates with SB contacts, and carriers are injected into the CNT channel via tunneling rather than via thermal excitation. It is not easy to obtain large current injection and high intensity light emission in such a p-n junction LED.

To obtain efficient light emission from CNT devices, we propose that large numbers of carriers can be injected from two electrodes at the same time without the SB, and they can then radiatively recombine with high efficiency. Our BFBD device provides a structure that can ensure two types of carriers (electrons and holes) are injected simultaneously [39]. EL measurements were also carried out in detail on our BFBD device, which is a true two-terminal light emitting diode (LED). Only one bias is needed for LED operation, and light emission can be observed under a low bias of $\sim 2 \mathrm{~V}$. Under a large forward bias, with the Sc contact being grounded, electrons can be injected, barrier-free, into the conduction band of the CNT from the Sc or Y contact. At the same time, holes can be injected into the valence band from the Pd electrode, as shown in Figure 5(c). The injected electrons and holes then recombine radiatively in the middle of the channel, appearing as a narrow emission peak with a FWHM of $\sim 30 \mathrm{meV}$. Complete EL spectroscopy measurements show that the emission process is dominated by radiative recombination of excitons.

Recently, extremely efficient light emission of the order of $10^{-4}$ photons per electron-hole pair in a $\mathrm{p}-\mathrm{n}$ diode based on CNTs was demonstrated with low current injection. However, this type of diode device relies on the use of at least three independent biases to drive the diode, to generate electron rich (n-) and hole rich (p-) regions, and is not a true two-terminal device. The device is also unsuitable for future integrated nanoelectronic and optoelectronic circuit applications, because of the increased complexity of the design and the fabrication process.

The EL spectrum of a typical BFBD device $(L=1 \mu \mathrm{m})$ in Figure 1(d) shows a clear emission peak at $0.925 \mathrm{eV}$, which may be well fitted using a Gaussian and corresponds to a peak with a FWHM of about $40 \mathrm{meV}$. The CNT used in 
(a)

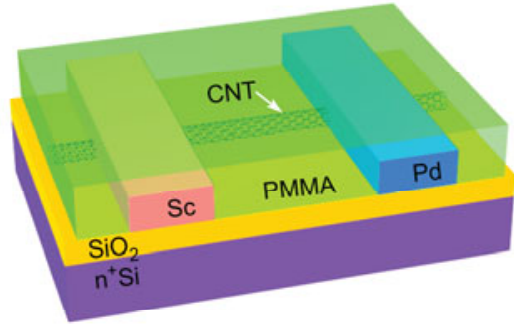

(c)

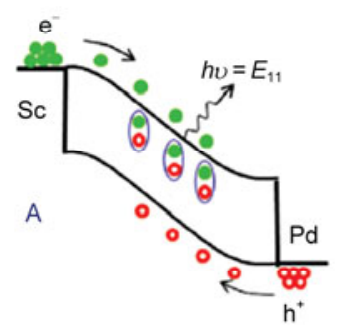

(b)

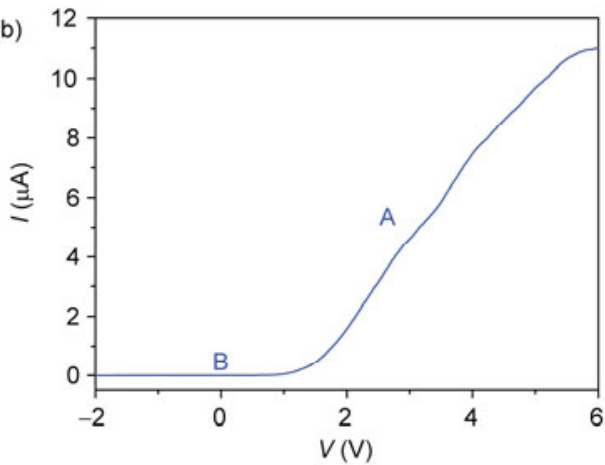

(d)

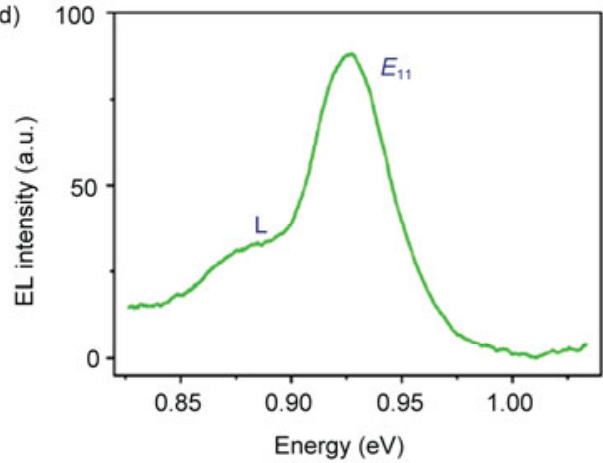

Figure 5 Structure and characteristics of a CNT LED with asymmetric contacts. (a) Schematic diagram illustrating the structure of an asymmetrically contacted CNT device. In all electrical measurements, the Sc contact was grounded and the bias V was applied to the Pd contact. (b) Experimental $I$ - $V$ characteristics of the CNT BFBD device with channel length $\sim 1 \mu \mathrm{m}$. (c) Band diagrams which correspond to the two representative points A (forward bias) and $\mathrm{B}$ (zero or small reverse bias) of (b). (d) EL spectrum of the diode with a large diode forward current $I=7.5 \mu \mathrm{A}$ for a CNT with $d \sim 1.14$ nm [39].

Figure 5 can be measured with Raman resonance spectra with a radial breathing mode (RBM) peak centred at $\Omega_{\mathrm{RBM}}$ $\approx 217 \mathrm{~cm}^{-1}$. The diameter of the CNT is found to be $\sim 1.14$ $\mathrm{nm}$ using the relation $d=248 / \Omega_{\mathrm{RBM}}[45,46]$. This is consistent with the $E_{1 \mathrm{u}}$ excitonic peak observed in our EL spectra, and also with photoluminescence (PL) excitation spectroscopy (Kataura plot) data for a CNT with $d \sim 1.14 \mathrm{~nm}$ [47-49]. The weaker emission peak marked by $L$ lower than the excitonic state in Figure 5(d) may be attributed to light emission from localized excitons associated with CNT defects [38].

The EL performance of the BFBD device shows typical current dependence. The EL intensity is seen to increase linearly with increasing injected current (Figure 6(b)). It should be noted that for this device, the FWHM of this emission peak at $0.925 \mathrm{eV}$ also increases with increasing current, from $26 \mathrm{meV}$ at $V=2.5 \mathrm{~V}$ to $33 \mathrm{meV}$ at $V=4.5 \mathrm{~V}$. This FWHM of the EL spectrum is very close to that observed in room temperature PL spectra, i.e. about $25 \mathrm{meV}$. The integrated EL intensity shows a highly linear relationship with current for two BFBD devices in the same SWCNT (Figure 6(b)). Our diode also exhibits a current threshold of $\sim 5 \mu \mathrm{A}$ for obvious infrared light emission.

The EL characteristics of the BFBD device depend on the gate voltage, and this provides extra control of the EL intensity via the gate bias. The gate transfer characteristics of the BFBD device (Figure 1(e)) show that the device is the same as an ambipolar FET with forward bias $V=1 \mathrm{~V}$, but all of the $I-V$ curves of the BFBD device show good rectifying behavior for all three gate voltages, $V_{\mathrm{g}}=-5,1$ and $5 \mathrm{~V}$. The main carriers are electrons or holes for large positive (e.g. $V_{\mathrm{g}}=5 \mathrm{~V}$ ) or negative gate voltage $\left(\right.$ e.g. $\left.V_{\mathrm{g}}=-5 \mathrm{~V}\right)$, respectively. Both EL spectra for these two gate voltages show the same dominant emission peak at $E \sim 0.925 \mathrm{eV}$, with a narrow FWHM of $\sim 30 \mathrm{meV}$. The gate voltage is mainly used to modulate the injection efficiency of electrons (or holes), as shown in Figure 6(d). At a large negative $V_{\mathrm{g}}=-5 \mathrm{~V}$, the hole is the majority carrier type injected into the CNT channel. These injected holes may recombine with electrons injected from the Sc contact via tunneling. However, the electron is the majority carrier type in the CNT channel with $V_{\mathrm{g}}=5 \mathrm{~V}$. The electrons injected from the Sc electrode may recombine with holes injected from the Pd contact via tunneling. Both cases yield a narrow peak in the EL spectra. Under these two conditions with the gate control, the EL processes are dominated by excitons formed near the electrode, the EL intensity is determined by the number of minority carriers, and the FWHM of the EL peak is therefore of the same magnitude of $\sim 30 \mathrm{meV}$.

We also compared the performance of the LED with that based on a unipolar n-type FET that is fabricated on the same SWCNT [39]. The external quantum efficiency of our BFBD device is shown to be more than three times higher than that of the FET based device. At the same time, the 

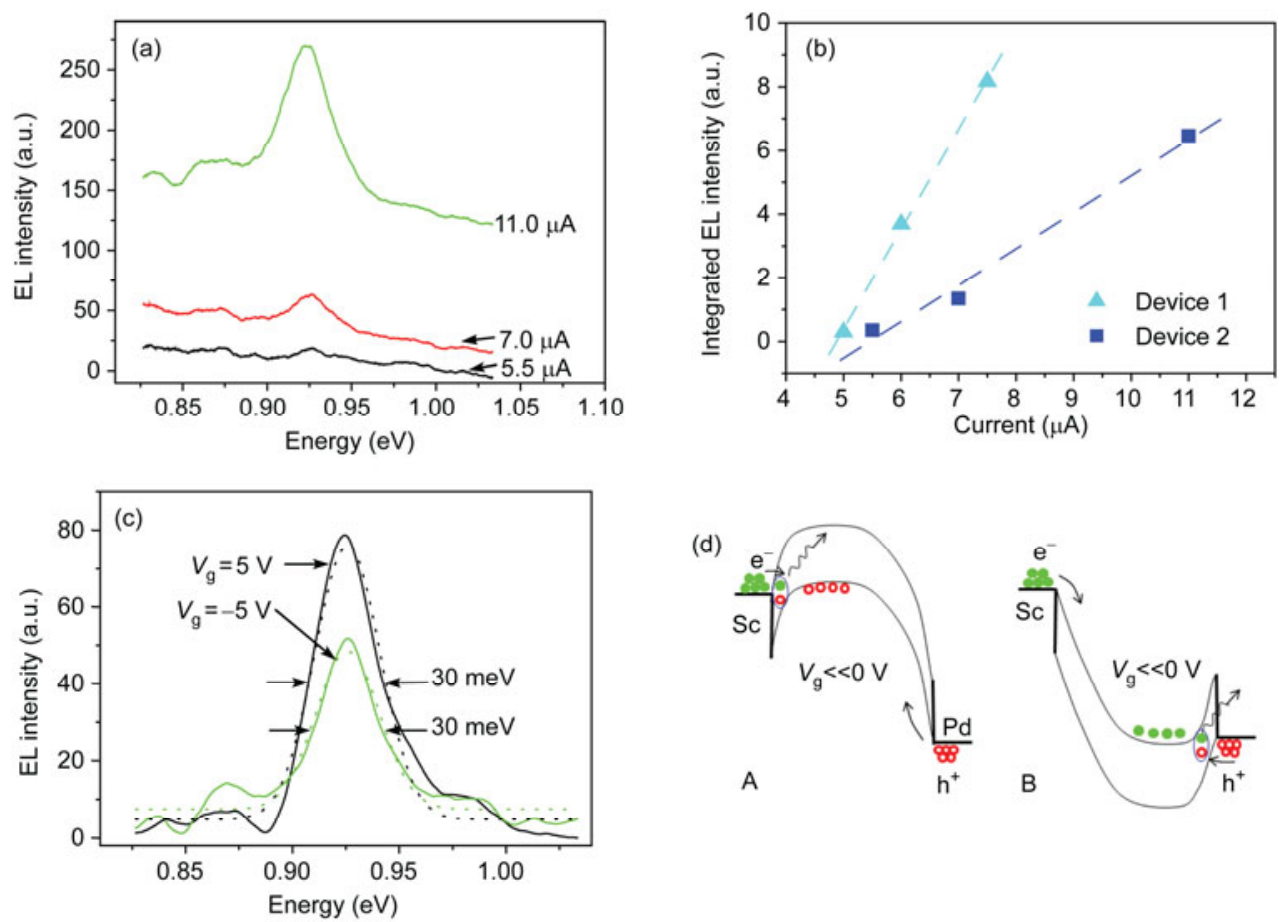

(d)

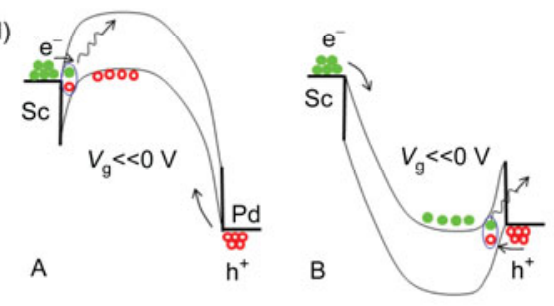

Figure 6 Current dependent EL spectrum and intensity of CNT BFBD device. (a) EL spectra obtained for currents between $5.5 \mu \mathrm{A}$ and $11 \mu \mathrm{A}$ for device 2 (fabricated on the same SWCNT as device 1, see Figure 5). The three EL spectra correspond to forward bias $V=2,2.5$ and 4.5 V. (b) Integrated emission intensity as a function of current for these two devices. (c) EL spectra of the device under two different currents, $I=8 \mu \mathrm{A}\left(V_{\mathrm{g}}=5 \mathrm{~V}\right)$ and $12 \mu \mathrm{A}\left(V_{\mathrm{g}}=-5 \mathrm{~V}\right)$. Both EL spectra can be fitted well using Gaussians with the same FWHM of $\sim 30 \mathrm{meV}$. (d) Band diagrams under different gate voltages. Points A and B correspond to $V_{\mathrm{g}}=-5 \mathrm{~V}$ and $V_{\mathrm{g}}=5 \mathrm{~V}$ of (c), respectively [39].

FWHM of the emission peak is $\sim 30 \mathrm{meV}$ in the LED spectrum, and is much narrower than that of the FET, at $\sim 150$ $\mathrm{meV}$. The operating voltage of the LED at $\sim 2 \mathrm{~V}$ is also lower than that of the FET at $\sim 10 \mathrm{~V}$.

\section{Summary and future work}

Carbon nanotubes can be used to construct photodetectors, solar cells and light emitters because of their excellent optical and electrical properties. Although great progress has been made in CNT optoelectronics, there are still some challenges ahead. One of these challenges is realization of a CNT-based nano laser. This will require us to improve the synthesis of single-type CNTs to produce enhanced identical light emission from arrays of CNTs.

Our doping-free fabrication of CNT based CMOS and optoelectronic devices thus provides an ideal platform for the future integration of CNT based nanoelectronic and optoelectronic devices. These CNT nano-scale light sources and detectors are also strong candidates for next generation inter-chip or on-chip optical communication.

This work was supported by the National Basic Research Program of China (2011CB933002, 2011CB933001), the Fundamental Research Funds for the Central Universities, and the National Natural Science Foundation of China (61071013, 61001016, 51072006, 60971003).
1 Avouris P, Freitag M, Perebeinos V. Carbon-nanotube photonics and optoelectronics. Nat Photonics, 2008, 2: 341-350

2 Durkop T, Getty S A, Cobas E, et al. Extraordinary mobility in semiconducting carbon nanotubes. Nano Lett, 2004, 4: 35-39

3 Prechtel L, Song L, Manus S, et al. Time-resolved picosecond photocurrents in contacted carbon nanotubes. Nano Lett, 2011, 11: 269-272

4 Kamat P V. Harvesting photons with carbon nanotubes. Nano Today, 2006, 1: 20-27

5 Zhu H W, Wei J Q, Wang K L, et al. Applications of carbon materials in photovoltaic solar cells. Sol Energy Mater Sol Cells, 2009, 93: 1461-1470

6 Dukovic G, Wang F, Song D, et al. Structural dependence of excitonic optical transitions and band-gap energies in carbon nanotubes. Nano Lett, 2005, 5: 2314-2318

7 Wang F, Dukovic G, Brus L E, et al. The optical resonances in carbon nanotubes arise from excitons. Science, 2005, 308: 838-841

8 Perebeinos V, Tersoff J, Avouris P. Scaling of excitons in carbon nanotubes. Phys Rev Lett, 2004, 92: 257402

9 Freitag M, Martin Y, Misewich J A, et al. Photoconductivity of single carbon nanotubes. Nano Lett, 2003, 3: 1067

10 Balasubramanian K, Fan Y, Burghard M, et al. Photoelectronic transport imaging of individual semiconducting carbon nanotubes. Appl Phys Lett, 2005, 87: 073101

11 Shim M, Siddons G P. Photoinduced conductivity changes in carbon nanotube transistors. Appl Phys Lett, 2003, 83: 3564

12 Avouris P, Afzali A, Appenzeller J, et al. Carbon nanotube electronics and optoelectronics. IEDM Tech Digest, 2004, 525

13 Lee J U. Photovoltaic effect in ideal carbon nanotube diodes. Appl Phys Lett, 2005, 87: 073101

14 Gabor N M, Zhong Z H, Bosnick K, et al. Extremely efficient multiple electron-hole pair generation in carbon nanotube photodiodes. Science, 2010, 325: 1367-1371 
15 Zhou C W, Kong J, Yenilmez E, et al. Modulated chemical doping of individual carbon nanotube. Science, 2000, 290: 1552-1555

16 Abdula D, Shim M. Performance and photovoltaic response of polymer-doped carbon nanotube p-n diodes. ACS Nano, 2008, 2: 2154

17 Lee J U, Gipp P P, Heller C M. Carbon nanotube p-n junction diodes. Appl Phys Lett, 2004, 85: 145-147

18 Shockley W, Queisser H. Detailed balance limit of efficiency of p-n junction solar cells. J Appl Phys, 1961, 32: 510-519

19 Wang S, Zhang Z Y, Ding L, et al. A doping-free carbon nanotube CMOS inverter-based bipolar diode and ambipolar transistor. Adv Mater, 2008, 20: 3258-3262

20 Javey A, Guo J, Wang Q, et al. Ballistic carbon nanotube field-effect transistors. Nature, 2003, 424: 654-657

21 Zhang Z Y, Liang X L, Wang S, et al. Doping-free fabrication of carbon nanotube based ballistic CMOS devices and circuits. Nano Lett, 2007, 7: 3603-3607

22 Zhang Z Y, Wang S, Ding L, et al. Self-aligned ballistic n-type singlewalled carbon nanotube field-effect transistors with adjustable threshold voltage. Nano Lett, 2008, 8: 3696-3701

23 Ding L, Wang S, Zhang Z Y, et al. Y-contacted high-performance n-type single-walled carbon nanotube field-effect transistors: Scaling and comparison with Sc-contacted devices. Nano Lett, 2009, 9: 4209-4214

24 Sze S M. Physics of Semiconductor Devices. New York: Wiley, 1981

25 Wang S, Zhang L H, Zhang Z Y, et al. Photovoltaic effects in asymmetrically contacted CNT barrier-free bipolar diode. J Phys Chem C, 2009, 113: 6891-6893

26 Chen C, Lu Y, Kong E S, et al. Nanowelded carbon-nanotube based solar microcells. Small, 2008, 4: 1313-1318

27 Wei J, Jia Y, Shu Q, et al. Double-walled carbon nanotube solar cells. Nano Lett, 2007, 7: 2317-2321

28 Jia Y, Wei J, Wang K, et al. Nanotube-silicon heterojunction solar cells. Adv Mater, 2008, 20: 4594-4598

29 Li Z, Kunets V, Saini V, et al. Light-harvesting using high density p-type single wall carbon nanotube/n-type silicon heterojunctions. ACS Nano, 2009, 3: 1407-1414

30 Zhang L, Jia Y, Wang S, et al. Carbon nanotube and CdSe nanobelt Schottky junction solar cells. Nano Lett, 2010, 10: 3583-3589

31 Liang C, Roth S. Electrical and optical transport of GaAs/carbon nanotube heterojunctions. Nano Lett, 2008, 8: 1809-1812

32 Brown P, Takechi K, Kamat P. Single-walled carbon nanotube scaffolds for dye-sensitized solar cells. J Phys Chem C, 2008, 112: 4776-4782

33 Robel I, Bunker B, Kamat P. Single-walled carbon nanotube-CdS nanocomposites as light-harvesting assemblies: Photoinduced chargetransfer interactions. Adv Mater, 2005, 17: 2458-2463
34 Misewich J A, Martel R, Avouris P, et al. Electrically induced optical emission from a carbon nanotube FET. Science, 2003, 300: 783-786

35 Chen J, Perebeinos V, Freitag M, et al. Bright infrared emission from electrically induced excitons in carbon nanotubes. Science, 2005, 310: 1171-1174

36 Marty L, Adam E, Albert L, et al. Exciton formation and annihilation during 1D impact excitation of carbon nanotubes. Phys Rev Lett, 2006, 96: 36803

37 Xia F, Steiner M, Lin Y M, et al. A microcavity-contralled, currentdriven, on-chip nanotube emitter at infrared wavelengths. Nat Nanotechnol, 2008, 3: 609-613

38 Mueller T, Kinoshita M, Steiner M, et al. Efficient narrow-band light emission from a single carbon p-n diode. Nat Nanotechnol, 2010, 5: 27-31

39 Wang S, Zeng Q S, Yang L J, et al. High-performance carbon nanotube light-emitting diodes with asymmetric contacts. Nano Lett, 2011, 11: 23-29

40 Adam E, Aguirre C M, Marty L, et al. Electroluminescence from single-wall carbon nanotube network transistors. Nano Lett, 2008, 8: 2351-2355

41 Lefebvre J, Austing D G, Finnie P. Two modes of electroluminescence from single-walled carbon nanotubes. Phys Status Solidi RRL, 2009, 3: 199-201

42 Engel M, Small J P, Steiner M, et al. Thin film nanotube transistors based on self-assembled, aligned, semiconducting carbon nanotube arrays. ACS Nano, 2008, 2: 2445-2452

43 Zaumseil J, Ho X, Guest J R, et al. Electroluminescence from electrolyte-gated carbon nanotube field-effect transistors. ACS Nano, 2009, 3: 2225-2234

44 Piper W W, Williams F E. Theory of electroluminescence. Phys Rev, 1958, 98: 1809-1813

45 Weisman R B, Bachilo S M. Dependence of optical energies on structure for single-walled carbon nanotubes in aqueous suspension: An empirical Kataura plot. Nano Lett, 2003, 3: 1235-1238

46 Bachilo S M, Strano M S, Kittrell C, et al. Structure-assigned optical spectra of single-walled carbon nanotubes. Science, 2002, 298: 23612366

47 O'Connell M J, Bachilo S M, Huffman C B, et al. Band gap fluorescence from individual single-walled carbon nanotubes. Science, 2002, 297: 593-596

48 Wang F, Dukovic G, Brus L E, et al. The optical resonances in carbon nanotubes arise from excitons. Science, 2005, 308: 838-841

49 Dukovic G, Wang F, Song D, et al. Structural dependence of excitonic optical transitions and band-gap energies in carbon nanotubes. Nano Lett, 2005, 5: 2314-2318

Open Access This article is distributed under the terms of the Creative Commons Attribution License which permits any use, distribution, and reproduction in any medium, provided the original author(s) and source are credited. 